\title{
Pharmacokinetic Characterization in Xenografted Mice of a Series of First-Generation Mimics for HLA-DR Antibody, Lym-1, as Carrier Molecules to Image and Treat Lymphoma
}

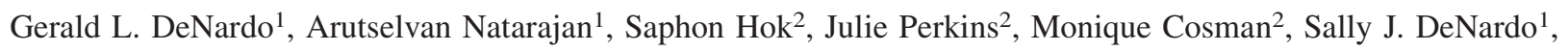
Felice C. Lightstone ${ }^{2}$, Gary R. Mirick ${ }^{1}$, Laird A. Miers ${ }^{1}$, and Rodney L. Balhorn ${ }^{2}$

${ }^{1}$ University of California Davis Cancer Center, Sacramento, California; and ${ }^{2}$ Lawrence Livermore National Laboratories, Livermore, California

If no one ever took risks, Michelangelo would have painted the Sistine floor.

Neil Simon

Despite their large size, antibodies (Abs) are suitable carriers to deliver systemic radiotherapy, often molecular image-based, for lymphoma and leukemia. Lym-1 Ab has proven to be an effective radioisotope carrier, even in small amounts, for targeting human leukocyte antigen DR (HLA-DR), a surface membrane protein overexpressed on B-cell lymphoma. Pairs of molecules (referred to as ligands), shown by computational and experimental methods to bind to each of 2 sites within the Lym-1 epitopic region, have been linked to generate small $(<2 \mathrm{kDa})$ molecules (referred to as selective high-affinity ligands [SHALs]) to mimic the targeting properties of Lym-1 Ab. Methods: A lysine-polyethylene glycol (PEG) backbone was used to synthetically link 2 of the following ligands: deoxycholate, 5-leuenkephalin, triiodothyronine, thyronine, dabsyl-L-valine, and $\mathrm{N}$-benzoyl-L-arginyl-4amino-benzoic acid to generate a series of 13 bidentate SHALs with a biotin or 1,4,7,10-tetraazacyclododecane- $N, N^{\prime}, N^{\prime \prime}, N^{\prime \prime \prime}-$ tetraacetic acid (DOTA) chelate attached to the linker. These SHALs have been assessed for their selectivity in binding to HLA-DR10-expressing cells and for their pharmacokinetics and tissue biodistribution in mice. Biotinylated versions of these SHALs discriminated cell lines positive for HLA-DR10 expression with near-nanomolar affinity. The DOTA versions of 4 SHALs were labeled with ${ }^{111}$ In for pharmacokinetic studies in mice with HLADR10-expressing malignant Raji xenografts. Results: The bidentate, biotinylated, and DOTA-SHALs were synthesized in high-purity, multimilligram amounts. Mean radiochemical and product yields and purities were $90 \%, 75 \%$, and $90 \%$ at mean specific activities of $3.9 \mathrm{MBq} / \mu \mathrm{g}(105 \mu \mathrm{Ci} / \mu \mathrm{g})$ for the ${ }^{111}$ In-labeled SHALs. As expected, rapid blood clearance and tumor targeting were observed. The pharmacokinetics of the SHALs was influenced by the component ligands. Biliary clearance, kidney localization, and serum receptor binding contributed to less favorable tumor targeting.

Received Feb. 21, 2007; revision accepted May 7, 2007.

For correspondence or reprints contact: Gerald L. DeNardo, MD, Radiodiagnosis \& Therapy, Molecular Cancer Institute, 1508 Alhambra Blvd., Room 3100, Sacramento, CA 95816.

E-mail: gldenardo@ucdavis.edu

COPYRIGHT @ 2007 by the Society of Nuclear Medicine, Inc.
Conclusion: A series of SHALs was readily synthesized in multimilligram amounts and showed the expected selective binding in vitro. Better selection of the SHAL components should provide second-generation SHALs with improved properties to fulfill the substantial potential of these novel molecular carriers for targeting.

Key Words: antibody; lymphoma; PET; imaging; therapy

J Nucl Med 2007; 48:1338-1347

DOI: 10.2967/jnumed.107.041095

I dentification of proteins unique to cancer cells and generation of antibodies (Abs) binding to them have led to improved management for non-Hodgkin's lymphoma (NHL). Monoclonal Ab (mAb) Lym-1, an attractive carrier of radioisotopes for imaging and therapy, binds to a human leukocyte antigen DR (HLA-DR) protein variant (1). HLADR has characteristics in common with other B-cell-surface antigens-for example, CD20-that make it an excellent target; disparate characteristics make it even more attractive. The basal level of HLA-DR expression on malignant B-cells is about 100 times more than that on normal B-cells $(2,3)$, so that only small amounts of Lym-1 Ab are needed to target extravascular malignant lymphoma $(4,5)$.

However, Abs are macromolecules that penetrate poorly and interact with a variety of $\mathrm{Fc}$ receptors, thereby limiting their selectivity as radioisotope carriers and adding to their adverse event profile. Furthermore, the immunogenicity of Ab-based reagents can be minimized, but not eliminated, using "humanized" Abs (6-10). In silico molecular modeling enables the design of small, multidentate molecules to bind to proteins. The mode of binding of these molecules mimics the specific, high-affinity interactions achieved through the formation of multiple contacts between residues on the surfaces of an $\mathrm{Ab}$ and its target (11-14). 
Although the development of multidentate high-affinity, target-specific ligands is a proven technology, it has not yet been widely applied to cancer imaging and therapy. We have characterized the Lym-1 epitope on HLA-DR, identified unique structural features within its epitope, and have developed a new class of small molecules - selective high-affinity ligands (SHALs) - to bind to malignant lymphocytes for molecular imaging and therapy. Linking together 2 ligands, each of which binds weakly to a protein and exhibits only modest selectivity, provides a molecule that binds to its target more tightly and with high selectivity (11-14). A series of bidentate "Ab mimics" for the epitopic region to which Lym-1 Ab binds to HLA-DR has been synthesized, using pairs of ligands shown to bind to sites within the Lym-1 epitopic region. These bidentate SHALs bind selectively to cultured human lymphoma cells with near-nanomolar affinity. They also bind to human NHL tissue, compete for binding with Lym-1, and do not bind to plasma proteins and other malignant cells that lack HLA-DR10 $(15,16)$. Because SHALs are much smaller than antibodies, these results suggest that SHALs can improve the imaging contrast and therapeutic index for NHL.

When compared with biologicals-for example, Abs and peptides-SHALs can be synthesized inexpensively in large amounts from commercially available components, have a long shelf life, and can be used in their initial form or as a precursor for creating more sophisticated targeting molecules. Here, we describe the pharmacokinetics and tissue biodistribution, in NHL xenografted mice, of a series of firstgeneration SHALs and the lessons learned that apply to their future development for molecular imaging and therapy.

\section{MATERIALS AND METHODS}

\section{Mouse Model}

Raji cells, human Burkitt's lymphoma cells obtained from the American Type Culture Collection (ATCC), were grown in RPMI 1640 and supplemented as recommended by the ATCC. Female athymic BALB/c nu/nu mice (Harlan Sprague-Dawley) were maintained according to the University of California animal care guidelines on a normal diet ad lib. At 6 wk of age, the mice received $4 \mathrm{~Gy}$ (400 rad) of external beam irradiation to suppress immune response to the Raji xenografts; $3-5$ d later, Raji cells $\left(6 \times 10^{6}\right)$ were injected subcutaneously on the abdomen of the mice. Implants grown 3 wk were measured in 3 orthogonal dimensions using a caliper; tumor volume was calculated using the formula for hemiellipsoids (17). At the time of SHAL injection, xenografts were well established. Mice with tumor volumes of $50-500 \mathrm{~mm}^{3}$ were used to evaluate the pharmacokinetics (17). A total of 112 mice were included in this study; there were between 20 and 42 mice in each group.

\section{SHAL Design and Chemistry}

Design. Computational docking was used to identify ligands that bind to either of 2 sites within the Lym-1 epitopic region. A program (SPHGEN) was used to identify potential ligand-binding sites on the surface of HLA-DR10 protein $(18,19)$; 3 unique sites were identified within the Lym-1 epitopic region. On the basis of their proximity to each other, the uniqueness of amino acid residues lining each site, and their location within the Lym-1 Ab region of

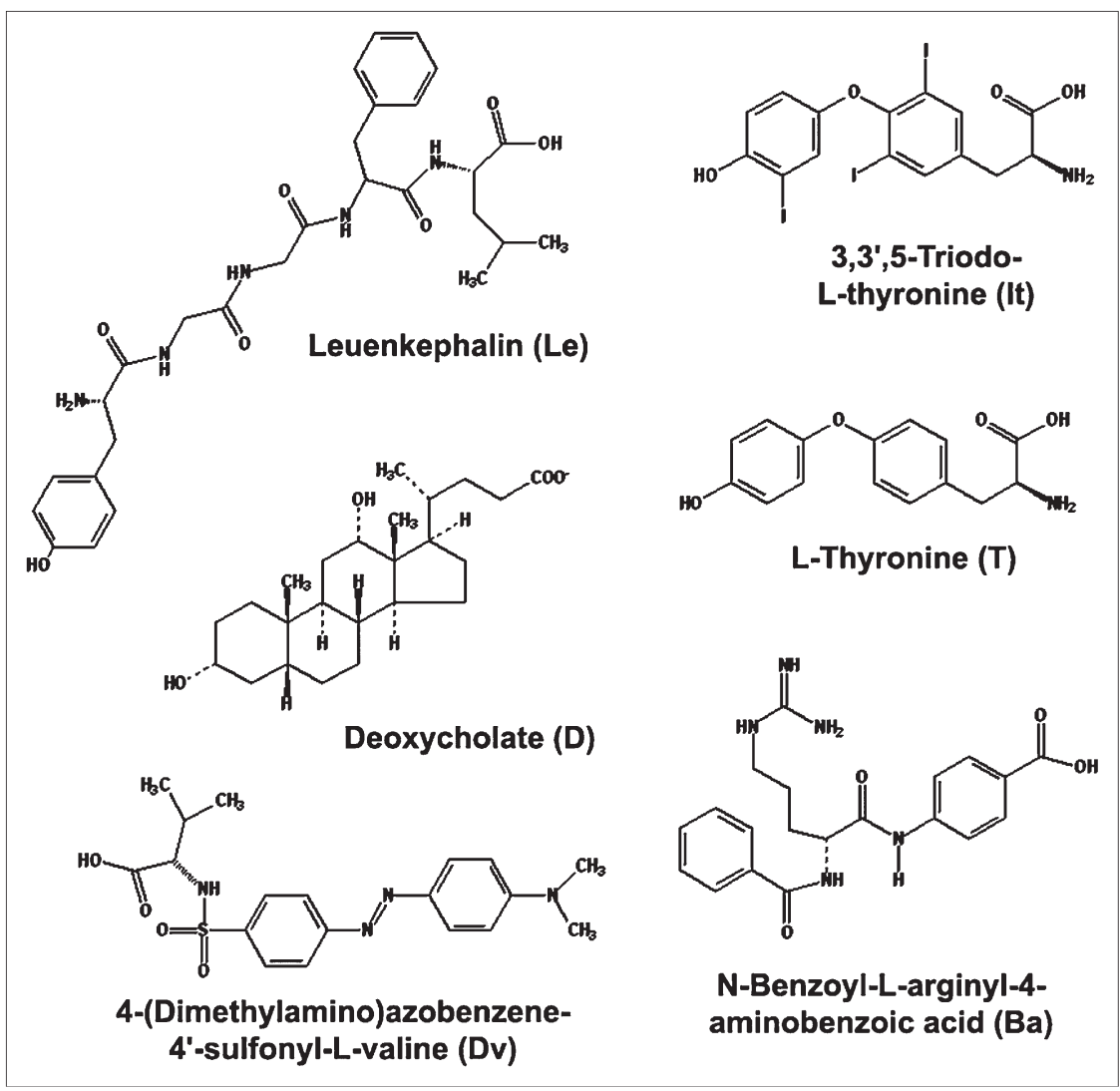

FIGURE 1. Chemical structures in 2 dimensions, and acronyms (in parentheses), for ligands used to generate SHALs. 
the HLA-DR protein, the 2 best sites were selected for virtual screening of the Available Chemicals Directory ([ACD] Molecular Design Limited, Inc.). Combinations of 2 ligands that were verified by nuclear magnetic resonance spectroscopy and surface plasmon resonance to bind to HLA-DR10 were used to synthesize SHALs.

Synthesis. SHALs to bind HLA-DR10, and related HLA-DRs, were synthesized using an orthogonally protected lysine residue of a solid-phase chlorotrityl chloride resin (16,20). All SHALs were based on a parent lysine molecule covalently bonded to various ligands. Briefly, the SHALs were synthesized by linking a site-1 ligand with a site-2 ligand using a lysine( $\mathbf{L})$-PEG(P)-based linker (PEG is polyethylene glycol) and a solid, polymer-supported synthesis on chlorotrityl chloride resin, as described (20). All of the ligands had carboxyl groups for ready conjugation to the amino groups on lysine. The ligand combinations used to synthesize SHALs were deoxycholate and leuenkephalin, deoxycholate and triiodothyronine, deoxycholate and thyronine, and dabsylvaline and $\mathrm{N}$-benzoyl-L-arginyl-4-aminobenzoic acid (Fig. 1). SHALs were synthesized with 0 , 1, or 2 short PEG linkers between the ligands to assess the effect of linker length.

The $\varepsilon$-amino group of the lysine was used to attach a biotin or $1,4,7,10$-tetraazacyclododecane- $N, N^{\prime}, N^{\prime \prime}, N^{\prime \prime \prime}$-tetraacetic acid (DOTA) chelate. Conversion to DOTA (or biotinylated) SHAL derivatives was accomplished by dissolving the SHALs in $500 \mu \mathrm{L}$ anhydrous $\mathrm{N}, \mathrm{N}$-dimethylformamide (DMF) and $100 \mu \mathrm{L}$ diisopropylethylamine. The biontinyl-OSu (341.39 g/mol, $1.1 \mathrm{eq})$ or hexafluorophosphate salt of DOTA $N$-hydroxy-succinimide ester $(933.36 \mathrm{~g} / \mathrm{mol}, 0.95-1.1 \mathrm{eq}$ ) was added to the mixture as a solid. The reaction was gently mixed for $15 \mathrm{~min}$ and monitored by analytic high-performance liquid chromatography (HPLC). On completion, the reaction solution was diluted with $300 \mu \mathrm{L} \mathrm{H}_{2} \mathrm{O}$ and $300 \mu \mathrm{L}$ acetonitrile, both containing $1 \%$ trifluoroacetic acid (TFA) and HPLC purified into two $600-\mu \mathrm{L}$ aliquots. Purified, lyophilized DOTA-SHALs were analyzed by HPLC and electrospray ionization mass spectrometry (ESI-MS).

The acronym for each SHAL consists of a 1- or 2-letter code for the ligand, with lysine and PEG designated as $\mathbf{L}$ and $\mathbf{P}$, respectively, and DOTA and biotin designated as Do and $\mathbf{B}$, respectively. Examples of SHALs that have been synthesized and their cor- responding identity and acronyms are shown in (Table 1; Fig. 2). SHALs were purified using reverse-phase (RP) HPLC. Analytic HPLC was performed at $1 \mathrm{~mL} / \mathrm{min}$ on an Agilent 1100 instrument using a Waters Symmetry C18, $5 \mu \mathrm{m}, 4.2 \times 150 \mathrm{~mm}$ column, and semipreparative HPLC was performed at $10 \mathrm{~mL} / \mathrm{min}$ on a Waters Symmetryprep C18, $7 \mu \mathrm{m}, 19 \times 300 \mathrm{~mm}$ column. Both HPLC methods used a linear gradient from $95 \% \mathrm{H}_{2} \mathrm{O}(1 \%$ TFA) to $80 \%$ MeCN (1\% TFA) over $12 \mathrm{~min}$ and a diode array for detection. SHALs were characterized using proton and carbon NMR spectroscopy and ESI-MS; all SHALs were found to have molecular weights within $0.07 \%$ of their theoretical molecular weight.

Radiochemistry. To determine the fraction of DOTAs available for metal chelation, each SHAL preparation was titrated using a cobalt assay (21). The concentration of the unchelated DOTASHAL was estimated using the HPLC peak area ultraviolet absorbance at $254 \mathrm{~nm}$. An aqueous solution of ${ }^{57} \mathrm{CoCl}_{2}$, calibrated by the manufacturer, was added to an aliquot of DOTA-SHAL in 10\% dimethyl sulfoxide (DMSO) in phosphate-buffered saline (PBS) diluted in $0.1 \mathrm{M} \mathrm{NH}_{4} \mathrm{OAc}, \mathrm{pH}$ 5.3. This mixture was adjusted to pH 7 using $\mathrm{NH}_{4} \mathrm{OAc}$ and held for $30 \mathrm{~min}$ at room temperature; ethylenediaminetetraacetic acid (EDTA; 0.1 M) was added to the reaction mixture, which was incubated for $15 \mathrm{~min}$ to sequester nonspecifically bound and excess cobalt ions. ${ }^{57} \mathrm{Co}$-DOTA-SHAL was separated using thin-layer chromatography (TLC) and precoated silica gel plates with $10 \%$ aqueous $\mathrm{NH}_{4} \mathrm{OAc} / \mathrm{MeOH}(1: 1$, $\mathrm{v} / \mathrm{v})$ as the eluent, and the concentration was determined by radiation counting using a 1282 CompuGamma CS Gamma Counter against a known quantity of the calibrated ${ }^{57} \mathrm{CoCl}_{2}$ solution. The metal chelatable DOTA was calculated as the ratio of the molar concentration of the ${ }^{57} \mathrm{Co}$-DOTA-SHAL chelate and the amount of DOTA-SHAL; this ratio ranged from $50 \%$ to $60 \%$ (Table 2).

DOTA-SHALs were labeled with carrier-free ${ }^{111} \mathrm{InCl}_{3}$ (MDS Nordion) using the following method (20). An aliquot of ${ }^{111} \mathrm{InCl}_{3}$ (15-20 $\mu \mathrm{L})$ was added to a solution of DOTA-SHAL $(25-50 \mu \mathrm{g})$ in $0.1 \mathrm{M} \mathrm{NH}_{4} \mathrm{OAc}$, pH $5.3(50 \mu \mathrm{L})$; the final $\mathrm{pH}$ of the reaction mixture was adjusted to 6.5 by adding $4 \mathrm{M} \mathrm{NH}_{4} \mathrm{OAc}$, and the mixture was incubated for $1 \mathrm{~h}$ at $37^{\circ} \mathrm{C}$. Then $0.1 \mathrm{M}$ EDTA $(10-20 \mu \mathrm{L})$

TABLE 1

List of DOTA- and Biotin- Bidentate SHALs

\begin{tabular}{|c|c|}
\hline Acronym & Identity \\
\hline \multicolumn{2}{|l|}{ DOTA-SHALS } \\
\hline LePLDDo & 5-Leuenkephalin PEG lysine deoxycholate DOTA \\
\hline LeacPLDDo & Acetylated 5-leuenkephalin PEG lysine deoxycholate DOTA \\
\hline ItPLDDo & Triiodothyronine PEG lysine deoxycholate DOTA \\
\hline DvLPTPLDo & Dabsyl-L-valine lysine PEG L-thyronine PEG lysine DOTA \\
\hline DvLPBaPLDo & Dabsyl-L-valine lysine PEG $N$-benzoyl-L-arginyl-4-amino-benzoic acid PEG lysine DOTA \\
\hline DVLPPBaPLDo & Dabsyl-L-valine lysine PEG PEG N-benzoyl-L-arginyl-4-amino-benzoic acid PEG lysine DOTA \\
\hline CtLPTPLDo & $\begin{array}{l}\text { 3-(2-([3-Chloro-5-trifluoromethyl)-2-pyridinyl]oxy)-anilino)-3-oxopropanionic acid lysine PEG L-thyronine PEG } \\
\text { lysine DOTA }\end{array}$ \\
\hline CtLPBaPLDo & $\begin{array}{l}\text { 3-(2-([3-Chloro-5-trifluoromethyl)-2-pyridinyl]oxy)-anilino)-3-oxopropanionic acid lysine } \\
\text { PEG N-benzoyl-L-arginyl-4-amino-benzoic acid PEG lysine DOTA }\end{array}$ \\
\hline \multicolumn{2}{|r|}{ 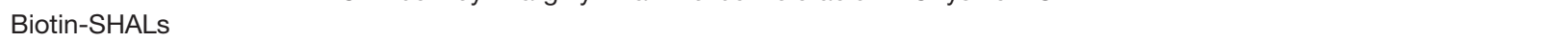 } \\
\hline LeLDB & 5-Leuenkephalin lysine deoxycholate biotin \\
\hline LePLDB & 5-Leuenkephalin PEG lysine deoxycholate biotin \\
\hline LeacPLDB & Acetylated 5-leuenkephalin PEG lysine deoxycholate biotin \\
\hline ItPLDB & Triiodothyronine PEG lysine deoxycholate biotin \\
\hline DVLPBaPLB & Dabsyl-L-valine lysine PEG N-benzoyl-L-arginyl-4-amino-benzoic acid PEG Iysine biotin \\
\hline
\end{tabular}




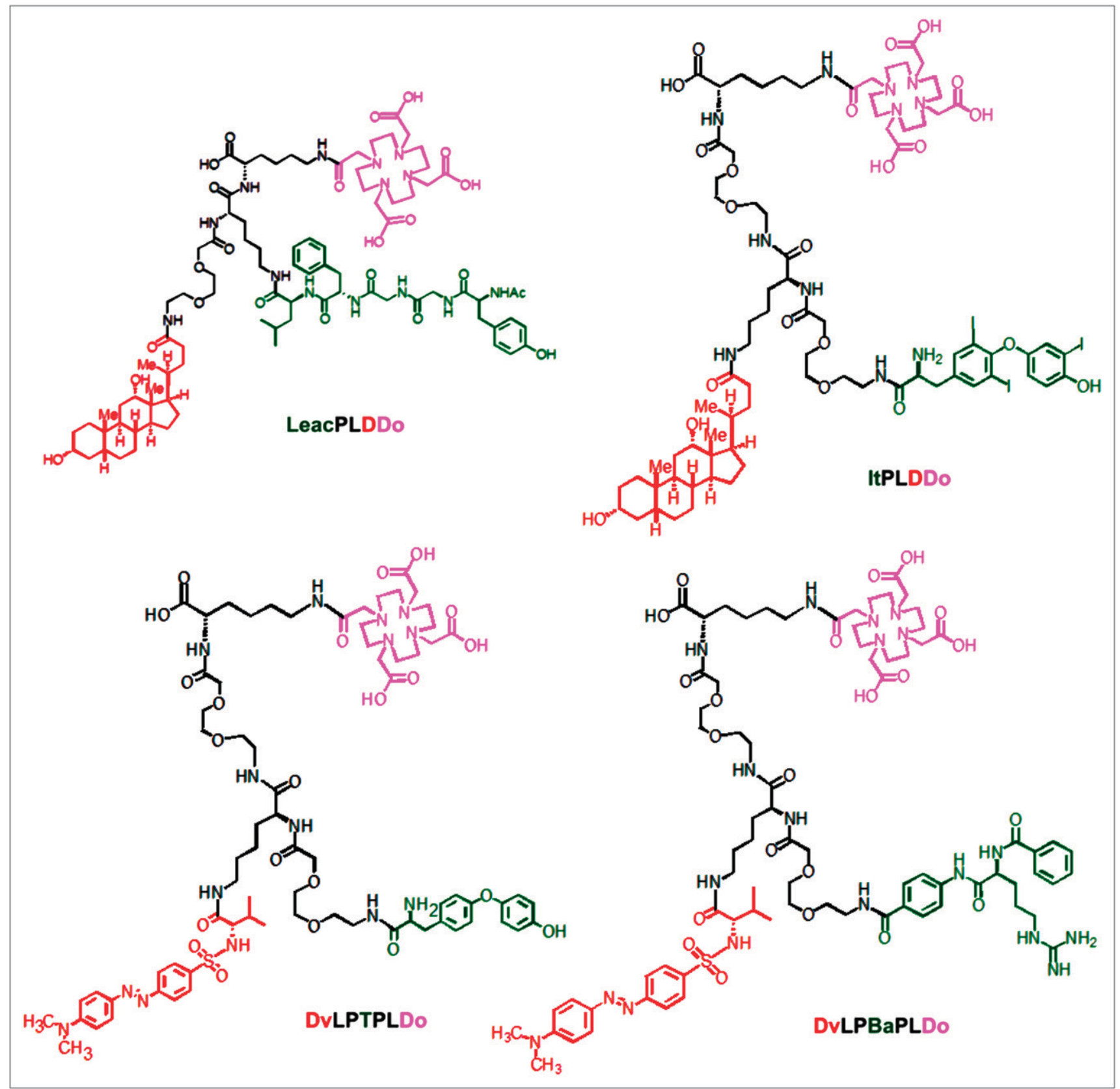

FIGURE 2. Chemical structures in 2 dimensions, and acronyms for 4 bidentate SHALs studied in mice: LeacPLDDo, acetylated 5-leuenkephalin PEG lysine deoxycholate DOTA; ItPLDDo, triiodothyronine PEG lysine deoxycholate DOTA; DvLPTPLDo, dabsylL-valine lysine PEG L-thyronine PEG lysine DOTA; and DvLPBaPLDo, dabsyl-L-valine lysine PEG N-benzoyl-L-arginyl-4aminobenzoic acid PEG lysine DOTA.

was added to sequester excess, free ${ }^{111} \mathrm{In}^{3+}$. The radiolabeled product was purified using HPLC, followed by dialysis in PBS with a $1-\mathrm{kDa}$ cutoff membrane. The purity of the radiolabeled SHALs was determined by TLC $\left(10 \% \mathrm{NH}_{4} \mathrm{OAc} / \mathrm{MeOH}, 1: 1\right)$, HPLC, and cellulose acetate electrophoresis (CAE). CAE resolved ${ }^{111}$ InDOTA-SHALs and ${ }^{111}$ In-EDTA; radioactive peaks were observed at $2.3-3.0 \mathrm{~cm}$ and $>6.5 \mathrm{~cm}$, respectively. Similar results were observed in the TLC assay; ${ }^{111}$ In-DOTA-SHALs showed little migration from the point of application $\left(\mathrm{R}_{\mathrm{f}}=0.25-0.3\right)$, whereas ${ }^{111}$ In-EDTA moved toward the solvent front $\left(\mathrm{R}_{\mathrm{f}}=0.5\right)$. By HPLC,

${ }^{111} \mathrm{In}$-EDTA eluted at 2.5-3.0 $\mathrm{mL}$ and ${ }^{111} \mathrm{In}$-DOTA-SHALs eluted at $9.5-10 \mathrm{~mL}$. The ${ }^{111} \mathrm{In}$ labeled SHALs were purified using RPHPLC (Fig. 3) or a 1-kDa dialysis membrane in PBS and concentrated using a Savant Speedvac SC110 (Thermo Fisher Scientific, Inc.). Final radiochemical purity was determined using C18 RPTLC (DC-Plastikfolien kieselgel 60 F254; EM Science), HPLC, and CAE. The final product was dissolved in 10\% DMSO in PBS and proved to be stable over $72 \mathrm{~h}$ at room temperature.

\section{Study Design}

Four bidentate ${ }^{111}$ In-DOTA-SHALs (LeacPLDDo, ItPLDDo, DvLPTPLDo, and DvLPBaPLDo) were studied in mice at 2 doses, 
TABLE 2

Data for 4 Bidentate DOTA-SHALs Evaluated in Mice

\begin{tabular}{lcc}
\hline \multicolumn{1}{c}{ Parameter } & Median & Range \\
\hline DOTAs available (\%) & 50 & $50-60$ \\
Radiochemical yield $(\%)$ & 90 & $75-95$ \\
Product yield (\%) & 75 & $50-90$ \\
Product purity (\%) & 90 & $80-97$ \\
Specific activity $(\mathrm{MBq} / \mathrm{nM})$ & 6.6 & $3.8-10.6$ \\
Specific activity $(\mathrm{MBq} / \mu \mathrm{g})$ & 3.9 & $2.1-5.3$
\end{tabular}

Data for 4 other bidentate DOTA-SHALs that were synthesized were similar.

1 or $20 \mu \mathrm{g}$. Pharmacokinetic investigations included serial biodistribution studies and whole-body autoradiography (WBAR). Mice were sacrificed at 2,4 , and $24 \mathrm{~h}$ after injection of ${ }^{111}$ In-DOTASHAL for biodistribution measurements and at 2 and $4 \mathrm{~h}$ after injection of ${ }^{111} \mathrm{In}$-DOTA-SHAL for WBAR.

\section{Pharmacokinetics}

The pharmacokinetics for each of the 4 SHALs were evaluated using serial biodistribution, WBAR, and blood studies. In groups of mice, each SHAL was injected into the tail vein in mass amounts of 1 or $20 \mu \mathrm{g}$ for biodistribution and $20 \mu \mathrm{g}$ for WBAR studies-1.1-1.9 MBq (30-50 $\mu \mathrm{Ci})$ and 7.4-11.1 MBq (200-300 $\mu \mathrm{Ci}$ ), respectively. Methods for obtaining pharmacokinetic data using ${ }^{111} \mathrm{In}$ have been described (22). The ${ }^{111} \mathrm{In}$ dose was measured using a CRC-12 dose calibrator (Capintec, Inc.) and was confirmed by counting the mouse immediately after injection, with 2 opposed, isoresponsive sodium iodide detectors (Picker Nuclear) calibrated for volume and geometry. Decay-corrected radioactivity was expressed as the percentage injected dose (\%ID) and $\% \mathrm{ID} / \mathrm{mL}$ or $\% \mathrm{ID} / \mathrm{g}$, respectively. To determine whole-body clearances, mice were counted immediately and serially after injection using the isoresponsive sodium iodide detector system; blood clearances were determined by collecting blood samples $(2 \mu \mathrm{L})$ from

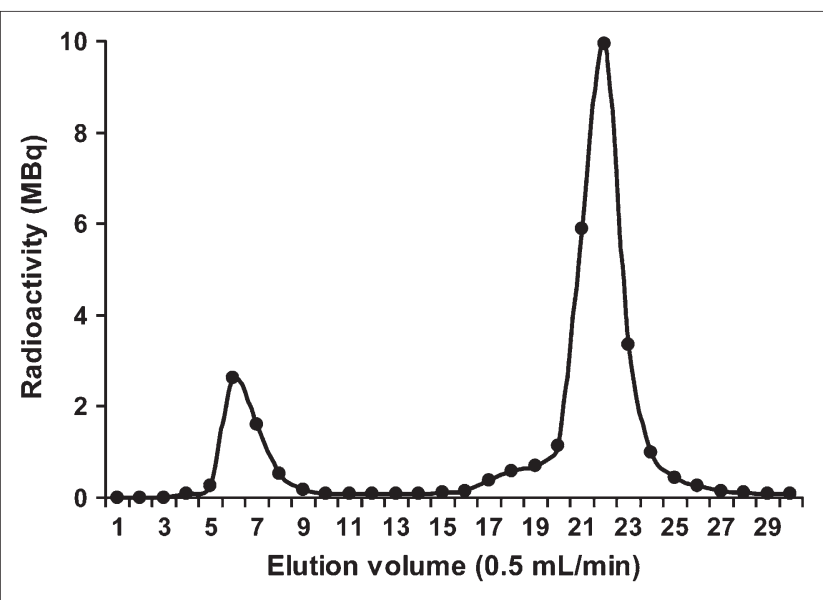

FIGURE 3. Purification of ${ }^{111}$ In-DOTA-SHAL (LeacPLDDo) reaction mixture was performed using $\mathrm{C} 18 \mathrm{RP}-\mathrm{HPLC}$ at 0.5 $\mathrm{mL} / \mathrm{min}$ (mobile phase, $\mathrm{CH}_{3} \mathrm{CN}$ and $\mathrm{H}_{2} \mathrm{O}$; gradient, $5 \%-90 \%$ $\mathrm{CH}_{3} \mathrm{CN}$ over $\left.30 \mathrm{~min}\right) .{ }^{111} \mathrm{In}$-DOTA-SHAL eluted at $21.3 \mathrm{~min}$ $(>95 \%)$; peak at 6.6 min corresponds to ${ }^{111} \mathrm{In}$-EDTA $(<5 \%)$. Purity of ${ }^{111} \mathrm{In}$-DOTA-SHAL was $>90 \%$. a tail vein and counting the samples in a sodium iodide $\gamma$-well counter (Packard). Decay-corrected radioactivity in the blood was expressed as \%ID, using a weight-based theoretic blood volume. These data over time were best fit to a monoexponential function. Pharmacokinetic data for other tissues were obtained by sacrificing mice at 2, 4, and $24 \mathrm{~h}$ after injection, removing and weighing the tissues, and counting them in the same $\gamma$-well counter. Using standards of comparable volume and geometry, the concentration in each sample was expressed as \% ID/g.

WBAR was conducted as previously described (23). Mice were anesthetized using an intravenous injection of $60 \mathrm{mg} / 100 \mu \mathrm{L}$ aqueous solution of sodium pentobarbital, which then were flash frozen in a hexane, dry ice bath. The frozen mice were embedded in $4 \%$ carboxymethylcellulose, and sagittal sections were generated with a Leica Polycut at $-20^{\circ} \mathrm{C}$. Sections of $50-\mu \mathrm{m}$ thickness were obtained to show tumors, spleen, kidney, liver, and the midline of the vertebral column. Radiographs of the sections were prepared by exposing the sections to x-ray film (BioMax MS; Kodak).

\section{Statistical Analysis}

Data are reported as mean $\pm \mathrm{SD}$. Statistical comparisons were based on the Wilcoxon rank sum test (24), a procedure based on ranking the values of 2 test groups. Differences were considered statistically significant if $P$ values $\leq 0.05$. Clearances were assessed using least-mean-square linear regression analyses of the $\%$ ID versus time for monoexponential and biexponential fits. Additionally, monoexponential fits for all mice in a group and only the mice that were followed for $24 \mathrm{~h}$ were compared and found to be insignificantly different. The $P$ values were determined by the transformation $Z=\mathrm{TANH}^{-1} r$ for the correlation coefficients (25).

\section{RESULTS}

\section{SHAL Chemistry}

Thirteen bidentate SHALs, 5 biotinylated and 8 DOTA conjugated containing 6 different ligands, have been synthesized in multimilligram amounts at high purity ( $\geq 99 \%)$, and the DOTA-SHALs labeled with ${ }^{111}$ In at high efficiency, specific activity, and purity (Table 2). All of the SHALs electrophoresed at or near the origin and eluted using molecular sieving chromatography following $12 \mathrm{~mL}$ of buffer, providing evidence that the DOTA-SHALs were nearly neutral and $<5 \mathrm{kDa}$ in size. Combinations of lysine and as many as 2 PEG monomers, used to vary the spacing between the ligands, did not alter binding in vitro (data not shown).

\section{Pharmacokinetics}

Although these studies were not designed to assess safety, no immediate toxicity was observed in the mice. For the bidentate SHALs (LeacPLDDo, ItPLDDo, DvLPTPLDo, and DvLPBaPLDo), the pharmacokinetics for 16 tissues were studied in groups of mice. Data are shown only for a subset of these tissues for practical reasons (Fig. 4). Pharmacokinetics did not vary for doses of SHAL of 1 and $20 \mu \mathrm{g}$. Both blood and whole-body clearances of the bidentate SHALs in the xenografted mice were best fitted with a monoexponential function. Blood clearance half-times varied between 3.9 and $7.1 \mathrm{~h}$, whereas whole-body clearance half-times varied between 5.5 and $20.1 \mathrm{~h}$ (Table 3; Fig. 4). In the mice, SHALs containing peptide ligands were directed to the kidney (Figs. 5 


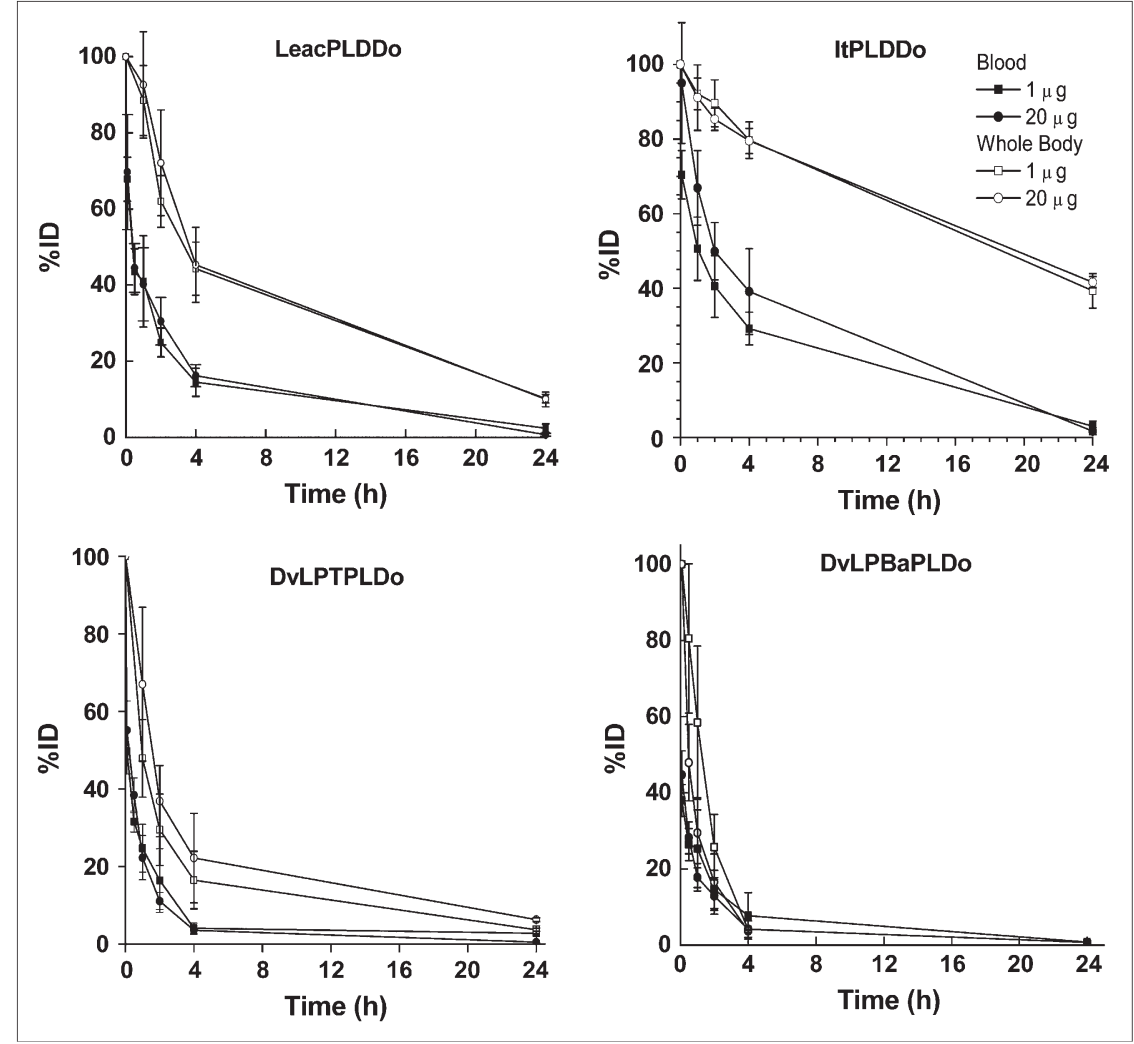

FIGURE 4. Whole-body and blood clearances of bidentate SHALs (LeacPLDDo, ItPLDDo, DvLPTPLDo, and DVLPBaPLDo) in mice. Clearances were similar whether 1 or $20 \mu \mathrm{g}$ of SHAL were given intravenously. Data are expressed as mean \pm SD. and 6). Others containing deoxycholate showed that this bile acid-like ligand resulted in substantial uptake by the liver. Modest uptake was seen in HLA-DR10-expressing Raji xenografts.

\section{DISCUSSION}

The incidence of NHL has increased at a rate of 3\%-4\% per year (26). New therapeutic options are needed because more than two thirds of NHL patients fail to achieve long-

TABLE 3

Blood and Whole-Body Clearances for Each of the 4 SHALs Given to Mice Intravenously in 1- or $20-\mu \mathrm{g}$ Doses

\begin{tabular}{|c|c|c|}
\hline \multirow[b]{2}{*}{ SHAL code } & \multicolumn{2}{|c|}{ Blood half-time $(\mathrm{h})$} \\
\hline & $1 \mu \mathrm{g}$ ( $P$ value $)$ & $20 \mu \mathrm{g}(P$ value $)$ \\
\hline LeacPLDDo & $5.7(0.3)$ & $3.9(<0.01)$ \\
\hline ItPLDDo & $5.5(0.01)$ & $4.3(<0.01)$ \\
\hline DVLPTPLDo & $7.1(0.16)$ & $4.0(0.06)$ \\
\hline \multirow[t]{3}{*}{ DvLPBaPLDo } & $4.6(0.02)$ & $4.7(0.05)$ \\
\hline & \multicolumn{2}{|c|}{ Whole-body half-time (h) } \\
\hline & $1 \mu \mathrm{g}$ ( $P$ value $)$ & $20 \mu \mathrm{g}(P$ value $)$ \\
\hline LeacPLDDo & $7.7(<0.01)$ & $7.5(0.01)$ \\
\hline ItPLDDo & $18.8(<0.01)$ & $20.1(<0.01)$ \\
\hline DvLPTPLDo & $6.3(0.01)$ & $7.1(0.05)$ \\
\hline DvLPBaPLDo & $5.5(0.02)$ & $6.1(0.05)$ \\
\hline
\end{tabular}

term, disease-free survival despite multiple drug chemotherapy $(27,28)$. Radioimmunotherapy has proven especially effective for NHL and compares favorably with other "salvage" therapies used to treat these patients; furthermore, toxicity is lower and patient acceptance is better (29-31). To improve on these results, we designed and synthesized a series of $\mathrm{Ab}$ mimics for the epitopic region of HLA-DR to which Lym-1 binds (2,3). This target was selected because Lym-1 $\mathrm{mAb}$ has proven to be a useful carrier molecule for therapy and imaging $(4,5)$. These bidentate SHALs are small molecules $(\leq 2 \mathrm{kDa}$ ) with associated advantages for molecular imaging and therapy. Because of their small size, they should readily penetrate vascular and tumor barriers and be excreted rapidly through the kidneys.

This series of bidentate SHALs was designed using the following rationale. A single ligand selected to bind to a site within the Lym-1 epitopic region of HLA-DR provides some selectivity but weak binding; the expectation was similar for a second ligand selected for another site within the Lym-1 epitopic region. When combined by a linker of length chosen for the distance between the 2 sites, selectivity and binding were expected to increase markedly $(15,32)$. The enhanced affinity and selectivity of a bidentate SHAL designed in this manner is derived from 3 factors: (i) the distance defined by the linker allows both ligands to bind simultaneously to the sites only if the sites are separated by the correct distance; (ii) the probability that both ligands simultaneously dissociate from the target protein 


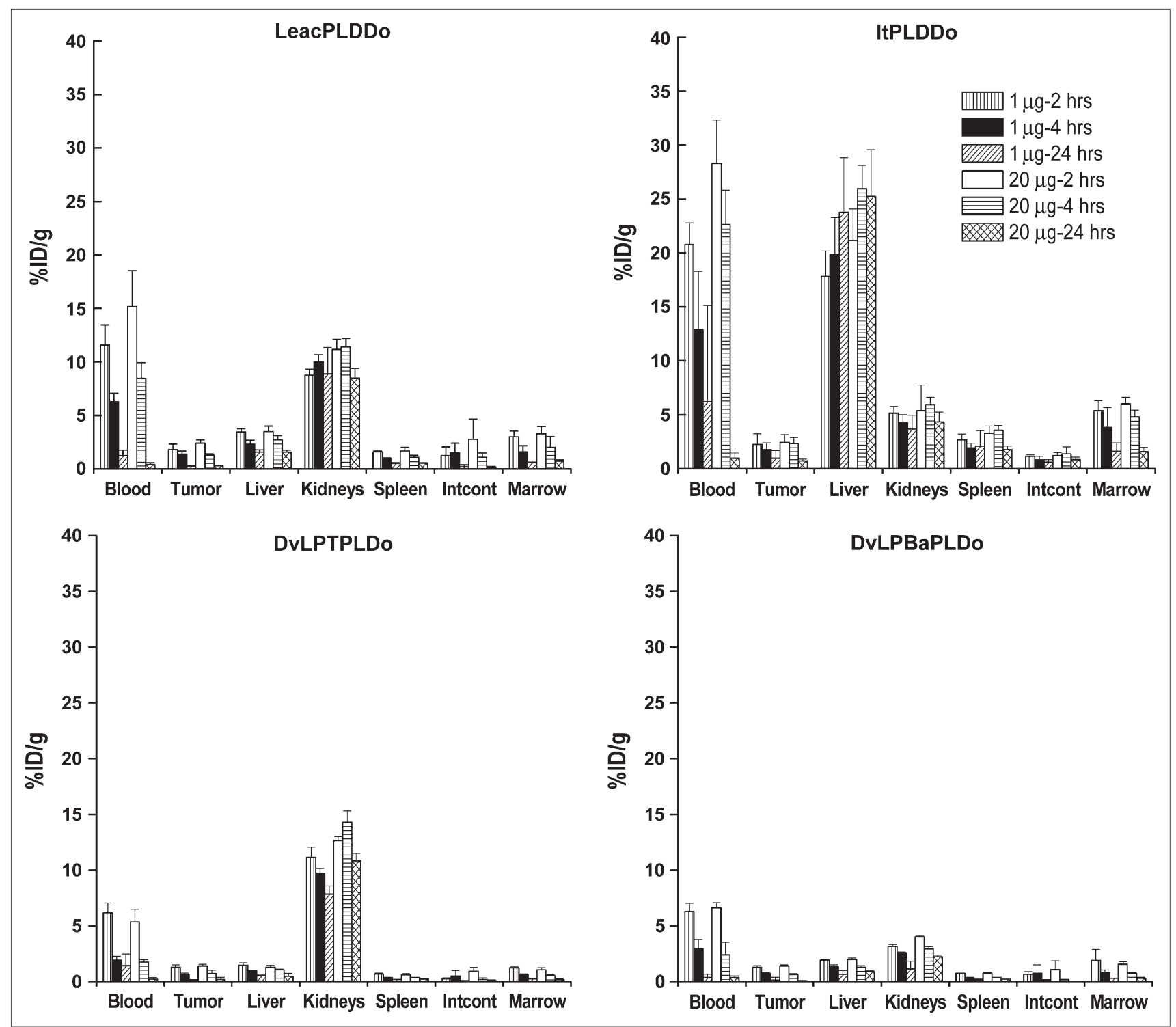

FIGURE 5. Tissue pharmacokinetics for bidentate SHALs in mice. Probably determined by the peptide ligands, leuenkephalin and triiodothyronine, and the deoxycholate ligand of the bidentate SHALs, respectively, there was kidney, liver, and intestinal content (Intcont) accumulation. SHAL, DvLPBaPLDo, in which dabsylvaline and aminobenzoic acid replaced the peptides and deoxycholate, had no accumulation in normal tissues. None of the SHALs showed a mass effect for their pharmacokinetics. Data are expressed as mean \pm SD.

is substantially lower than the probability that only one will release; and, (iii) the linker prevents a dissociated ligand from diffusing away from the protein. If either ligand of the SHAL binds independently to another protein, the ligand dissociates rapidly because of its weak affinity. Thus, the bidentate SHAL binds tightly to a protein only when both ligands bind simultaneously to the protein. It is highly improbable that identical sites separated by the same distance exist on proteins other than the target protein for which the SHAL was designed.

Thirteen bidentate SHALs, consisting of 2 of 6 different ligands identified to bind to either of 2 sites within the Lym-1 epitopic region, have been synthesized using a combination of lysine and PEG monomers to create the linker between the ligands. One unique feature of the synthetic scheme used to create the SHALs is that the approach allowed the attachment of biotin to the linker for in vitro assays or DOTA chelate for animal studies. Multimilligram amounts of pure SHALs having a biotin or DOTA attached to the linker were readily synthesized. Biotin made it possible to assess the binding of the SHALs to isolated HLA-DR10 protein by surface plasmon resonance and to examine their selectivity for HLA-DR-expressing live cells and tissue sections. These biotinylated, first-generation SHALs bound to HLA-DR10 protein and HLA-DR10-expressing NHL cells with nearnanomolar affinity (but not cells lacking HLA-DR10) and to sections of human lymphoma tissue (15). The selectivity of the biotinylated SHALs for HLA-DR-expressing cells 


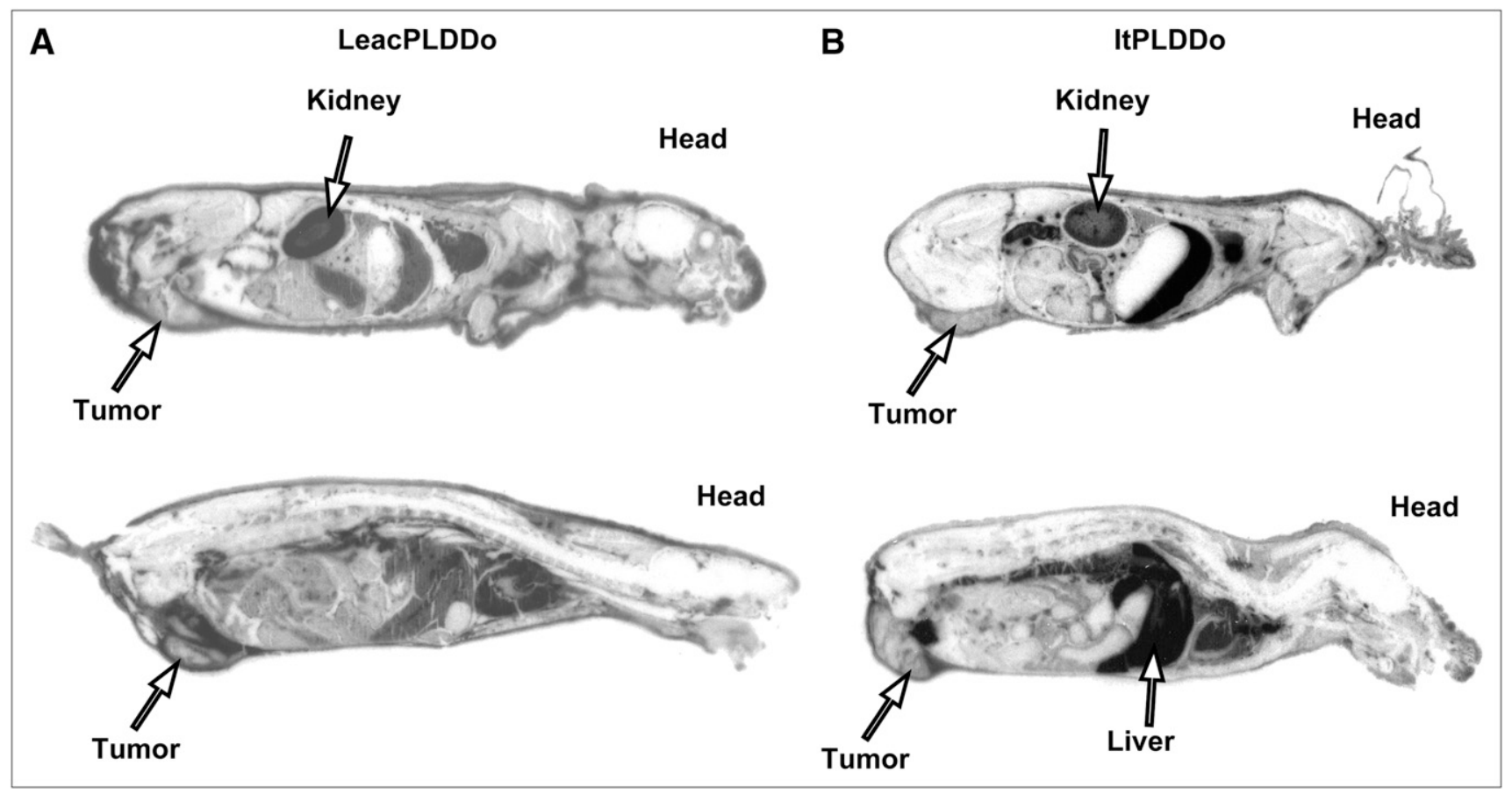

FIGURE 6. WBARs of mice euthanized $2 \mathrm{~h}$ (top) and $4 \mathrm{~h}$ (bottom) after intravenous injection of $20 \mu \mathrm{g}$ of $111 \mathrm{ln}$ bidentate deoxycholate-leuenkephalin (A) and deoxycholate-triiodothyronine (B) SHALs. These SHALs showed kidney and liver localization, respectively.

was improved by assay formats in which streptavidin converted the bidentate SHALs to multivalent species (data not shown).

Once a bidentate SHAL was confirmed to be selective in vitro, a corresponding SHAL with DOTA replacing the biotin on the linker was synthesized to enable the use of radiometals for imaging and therapeutic applications. DOTASHALs were readily labeled with ${ }^{111}$ In at high efficiency, specific activity, and purity suitable for studies in animals. For the first generation of SHALs, blood clearance was rapid, whereas body clearance was variable, depending on the ligands. Some SHALs accumulated in the liver or the kidney, demonstrating the importance of biologic considerations for ligand selection. The kidney and liver specificities of the SHALs appear to reflect the binding preferences of the individual ligands that made up these initial SHALs. This motivated us to exchange dabsylvaline for deoxycholate for site 1 and to replace leuenkephalin or triiodothyronine with benzoyl-L-arginyl-4-aminobenzoic acid for site 2. This SHAL, DvLPBaPLDo, exhibited more promising results in mice. Exchanging dabsylvaline for deoxycholate eliminated localization in the liver. Replacement of peptide-like ligands eliminated kidney uptake. Blood, body, and normal tissue clearances of this SHAL were rapid, as desired. However, xenograft clearance of this SHAL was also rapid, indicating that the off-rate of the bound SHAL needs to be improved.

This series of SHALs has shown impressive selectivity in vitro and near-nanomolar binding affinity. The length of the PEG linker for the ligands was selected on the basis of the modeled distance between the sites. Although the linker length between the ligands was assumed to be critical, linkers consisting of lysine alone or lysine with 1 or 2 PEG monomers seemed to bind to HLA-DR equally well. The results provided by these first SHAL pharmacokinetic studies, conducted in mice bearing lymphoma xenografts, have led to specific changes that improved their performance in vivo and guide additional changes to improve their effectiveness. When biologic considerations were included in the ligand selection process, the resulting SHAL, DvLPBaPLDo, showed no localization in, and rapid clearance from, normal tissues. Modest NHL tumor accumulation and the rapid kinetics of this bidentate SHAL suggest that it has potential for imaging NHL. However, the residence time of this SHAL in HLA-DR-expressing malignant Raji xenografts was not long enough to consider this SHAL for therapy. Like scFv $\mathrm{Ab}$ fragments, the binding affinity of the bidentate SHALs synthesized to date was not strong enough to provide prolonged tumor residence times.

To improve their potential for therapy, the SHAL affinity must be increased. This can be achieved by either of 2 approaches designed to increase the interactions between the SHAL and its protein target. One approach is to generate multidentate SHALs having more binding regions beyond those of a bidentate SHAL, thereby increasing both the affinity and the selectivity. Another approach is to generate dimeric (bis), or even trimeric, SHALs to emulate the multivalent binding of an $\mathrm{Ab}$ (33). SHALs of both types continue to be very small as they have molecular weights of $<5 \mathrm{kDa}$. Consequently, rapid target penetration and localization, and normal tissue clearance, needed for high contrast in 

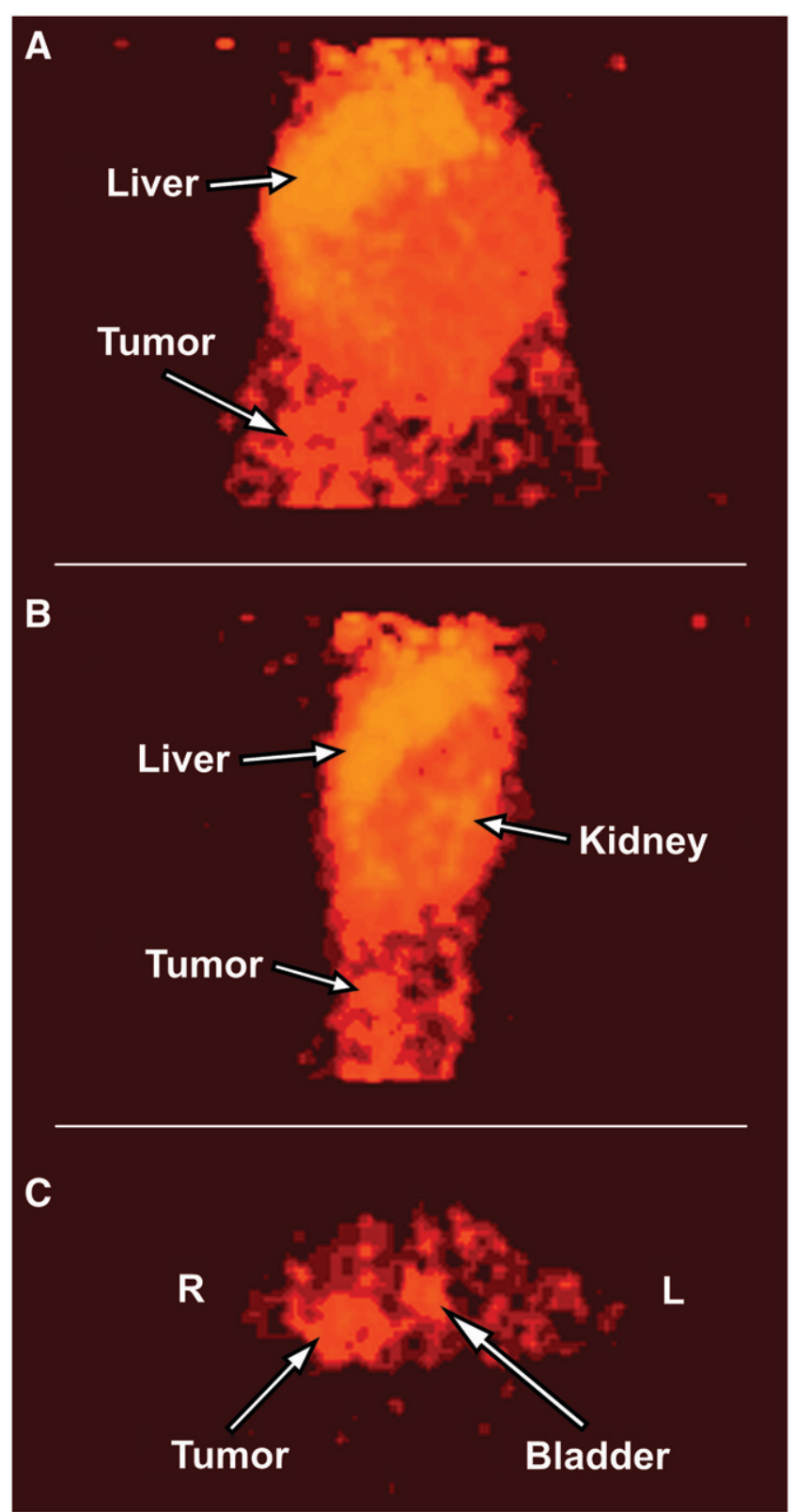

FIGURE 7. To assess SHAL potential for molecular imaging, small-animal PET tomographs of a mouse in the prone position were obtained $2 \mathrm{~h}$ after intravenous injection of ${ }^{64} \mathrm{Cu}$-labeled LePLDDo (7.4 MBq, $210 \mu \mathrm{Ci} ; 4 \mu \mathrm{g})$. Coronal (A), sagittal (B), and transverse $(C)$ sections show increased radioactivity in liver, kidney, bladder, and Raji human NHL, but not in the HBT 3477 human breast cancer xenografts on the right and left lower abdomen, respectively. A Raji xenograft was established subcutaneously in the right lower abdomen because these cells express HLA-DR10 (1) and an HBT 3477 xenograft was established subcutaneously in the left lower abdomen because these cells do not express HLA-DR10 (36). These xenografts provided positive (Raji) and negative (HBT 3477) controls for the epitopic region of HLA-DR10 to which Lym-1 Ab binds and for which the SHALs were designed to target. microPET scans were performed on a Focus120 system (Siemens Medical Solutions USA, Inc.) and analyzed with ASIPro and the Maximum A Posteriori iterative algorithm. The mouse was anesthetized using isoflurane. molecular imaging and high therapeutic indices in molecular therapy, are preserved.

The SHAL platform is especially attractive for molecular imaging and radiotherapy. The SHALs are easily conjugated to DOTA, a chelator suitable for a variety of metals (34), including gadolinium for MRI, ${ }^{111}$ In for nuclear imaging, ${ }^{64} \mathrm{Cu}$ for positron tomographic imaging (Fig. 7), and therapeutic radiometals such as copper-67 and the radiolanthanides. Furthermore, the lysine in the linker provides a site for ${ }^{18}$ F-FDG PET using established chemistry (35).

\section{CONCLUSION}

In conclusion, SHALs have been synthesized in multimilligram amounts suitable for efficient radiometal labeling at high product yield and purity. When compared with Abs and peptides, SHALs have a shelf-life measured in years, an availability above, and cost below, that of these biologic materials, and potential for oral administration. Although individual ligands may unfavorably direct the tissue distribution of a SHAL, SHALs produced by swapping out unfavorable ligands showed markedly improved tissue distribution. Importantly, the SHAL technology established for HLA-DR can be transferred to any other protein target of interest.

\section{ACKNOWLEDGMENTS}

This research was supported by National Cancer Institute grant PO1-CA47829 and Lawrence Livermore National Laboratory LDRD awards 01-ERD-111, 01-ERD-046, and 01-SI-012, under the auspices of U.S. Department of Energy contract W-7405-ENG-48. We thank Aina Yuan and ChengYi Xiong for assistance in pharmacokinetic data assembly, Christine Hartmann-Siantar for guidance, Vladimir Sysko for assistance with data assembly and manuscript preparation, and Julie Sutcliffe-Goulden, PhD, and Simon Cherry, $\mathrm{PhD}$, for generating the small-animal PET data for Figure 7.

\section{REFERENCES}

1. Epstein AL, Marder RJ, Winter JN, et al. Two new monoclonal antibodies, Lym-1 and Lym-2, reactive with human-B-lymphocytes and derived tumors, with immunodiagnostic and immunotherapeutic potential. Cancer Res. 1987;47: 830-840.

2. Rose LM, Deng CT, Scott S, et al. Critical Lym-1 binding residues on polymorphic HLA-DR molecules. Mol Immunol. 1999;36:789-797.

3. Rose LM, Gunasekera AH, DeNardo SJ, DeNardo GL, Meares CF. Lymphomaselective antibody Lym-1 recognizes a discontinuous epitope on the light chain of HLA-DR10. Cancer Immunol Immunother. 1996;43:26-30.

4. DeNardo GL, DeNardo SJ, Goldstein DS, et al. Maximum tolerated dose, toxicity, and efficacy of ${ }^{131} \mathrm{I}$-Lym-1 antibody for fractionated radioimmunotherapy of non-Hodgkin's lymphoma. J Clin Oncol. 1998;16:3246-3256.

5. DeNardo GL, DeNardo SJ, Lamborn KR, et al. Low-dose fractionated radioimmunotherapy for B-cell malignancies using ${ }^{131} \mathrm{I}-\mathrm{Lym}-1$ antibody. Cancer Biother Radiopharm. 1998;13:239-254

6. Brown KS, Levitt DJ, Shannon M, Link BK. Phase II trial of Remitogen (humanized 1D10) monoclonal antibody targeting class II in patients with relapsed low-grade or follicular lymphoma. Clin Lymphoma. 2001;2:188-190.

7. Kostelny SA, Link BK, Tso JY, et al. Humanization and characterization of the anti-HLA-DR antibody 1D10. Int J Cancer. 2001;93:556-565. 
8. Leonard JP, Link BK. Immunotherapy of non-Hodgkin's lymphoma with hLL2 (epratuzumab, an anti-CD22 monoclonal antibody) and Hu1D10 (apolizumab). Semin Oncol. 2002;29(1 suppl 2):81-86.

9. Ligibel JA, Winer EP. Trastuzumab/chemotherapy combinations in metastatic breast cancer. Semin Oncol. 2002;29:38-43.

10. Lundin J, Kimby E, Bjorkholm M, et al. Phase II trial of subcutaneous anti-CD52 monoclonal antibody alemtuzumab (Campath- ${ }^{1} \mathrm{H}$ ) as first-line treatment for patients with B-cell chronic lymphocytic leukemia (B-CLL). Blood. 2002;100: $768-773$.

11. Fan E, Merritt EA, Verlinde CL, Hold WG. AB(5) toxins: structures and inhibitor design. Curr Opin Struct Biol. 2000;10:680-686.

12. Kramer RH, Karpen JW. Spanning binding sites on allosteric proteins with polymer-linked ligand dimers. Nature. 1998;395:710-713.

13. Hajduk PJ, Meadows RP, Fesik SW. Discovering high-affinity ligands for proteins. Science. 1997;278:497-499.

14. Mammen M, Choi S, Whitesides GM. Polyvalent interactions in biological systems: implications for design and use of multivalent ligands and inhibitors. Agnew Chem Int Edit. 1998;37:2754-2794.

15. Balhorn R, Hok S, Burke P, et al. Selective high affinity ligand (SHAL) "antibody mimics" for cancer diagnosis and therapy: initial application to lymphoma/ leukemia. Clin Cancer Res. In press.

16. West J, Perkins J, Hok S, et al. Direct antilymphoma activity of novel, firstgeneration "antibody mimics" that bind HLA-DR10-positive non-Hodgkin's lymphoma cells. Cancer Biother Radiopharm. 2006;21:645-654.

17. DeNardo GL, Kukis DL, Shen S, et al. Efficacy and toxicity of ${ }^{67} \mathrm{Cu}-2$ IT-BATLym-1 radioimmunoconjugate in mice implanted with Burkitt's lymphoma (Raji). Clin Cancer Res. 1997;3:71-79.

18. Kuntz ID, Blaney JM, Oatley SJ, Langridge R, Ferrin TE. A geometric approach to macromolecule-ligand interactions. J Mol Biol. 1982;161:269-288.

19. Desjarlais RL, Sheridan RP, Seibel GL, Dixon JS, Kuntz ID, Venkataraqhavan R. Using shape complementarity as an initial screen in designing ligands for a receptor binding site of known three-dimensional structure. J Med Chem. 1988; 31:722-729.

20. Hok S, Natarajan A, Balhorn R, DeNardo SJ, DeNardo GL, Perkins J. Synthesis and radiolabeling of selective high affinity ligands designed to target nonHodgkin's lymphoma and leukemia. Bioconjug Chem. 2007;18:912-921.

21. Kukis DL, DeNardo GL, DeNardo SJ, et al. Effect of the extent of chelate substitution on the immunoreactivity and biodistribution of 2IT-BAT-Lym-1 immunoconjugates. Cancer Res. 1995;55:878-884.
22. DeNardo GL, DeNardo SJ, Peterson JK, et al. Preclinical evaluation of cathepsindegradable peptide linkers for radioimmunoconjugates. Clin Cancer Res. 2003;9: 3865 s-3872s.

23. Fand I, McNally WP. The technique of whole-body autoradiography. In: Johnson JE, ed. Current Trends in Morphological Techniques. 1st ed. Boca Raton, FL: CRC Press; 1981:1-28.

24. Hollander M, Wolfe DA. Nonparametric Statistical Methods. New York, NY: Wiley; 1973.

25. Beyer WH, ed. CRC Handbook of Tables for Probabilities and Statistics. 2nd ed. Boca Raton, FL: CRC Press; 1968.

26. Devesa SS, Fears T. Non-Hodgkin's lymphoma time trends: United States and international data. Cancer Res. 1992;52:5432s-5440s.

27. Armitage JO. Treatment of non-Hodgkin's lymphoma. N Engl J Med. 1993;328: 1023-1030.

28. Fisher RI, Gaynor ER, Dahlberg S, et al. Comparison of a standard regimen (CHOP) with three intensive chemotherapy regimens for advanced non-Hodgkin's lymphoma. N Engl J Med. 1993;328:1002-1006.

29. DeNardo GL, Sysko VV, DeNardo SJ. Cure of incurable lymphoma. Int J Radiat Oncol Biol Phys. 2006;66(2):s46-s56.

30. Witzig TE, White CA, Wiseman GA, et al. Phase I/II trial of IDEC-Y2B8 radioimmunotherapy for treatment of relapsed or refractory CD20+ B-cell nonHodgkin's lymphoma. J Clin Oncol. 1999;17:3793-3803.

31. Witzig TE, White CA, Gordon LI. Safety of yttrium-90 ibritumomab tiuxetan radioimmunotherapy for relapsed low-grade, follicular, or transformed nonHodgkin's lymphoma. J Clin Oncol. 2003;21:1263-1270.

32. Balhorn R, Perkins SJ, Burke PA, et al. Development of selective high affinity ligand (SHAL) antibody mimics for lymphoma/leukemia imaging and therapy [abstract]. Cancer Biother Radiopharm. 2004;19(4):517.

33. Adams GP, Tai MS, McCartney JE, et al. Avidity-mediated enhancement of in vivo tumor targeting by single-chain Fv dimers. Clin Cancer Res. 2006;12: 1599-1605.

34. Meares CF, Diril H, Kukis DL, et al. Radiochemistry of antibodies: some recent advances. Antibody Immunoconj Radiopharm. 1991;4:389-398.

35. Sutcliffe-Goulden JL, O’Doherty MJ, Marsden PK, Hart IR, Marshall JF, Bansal SS. Rapid solid phase synthesis and biodistribution of ${ }^{18} \mathrm{~F}$-labelled linear peptides. Eur J Nucl Med Mol Imaging. 2002;29:754-759.

36. Winthrop MD, DeNardo SJ, Muenzer JT, Chi SG, Gumerlock PH. p53independent response of a human breast cancer xenograft to radioimmunotherapy. Cancer. 1997;80(suppl 12):2529-2537. 